Results: At the end of mud-bath therapy, using first a semi-quantitative approach, we observed in both biological replicates increased levels ( $>1.5$ fold change) of: inhibin beta A subunit (INHBA), activin A receptor type 2B (ACVR2B), angiopoietin-1 (ANGPT1), beta-2-microglobulin (B2M), growth differentiation factor 10 (BMP-3b/GDF10), C-X-C motif chemokine ligand 5 (CXCL5), fibroblast growth factor 2 (FGF2), fibroblast growth factor 12 (FGF12), oxidized low density lipoprotein receptor 1 (OLR1), matrix metallopeptidase 13 (MMP13). We observed that some increased proteins belongs to the same family or pathway: INHBA, ACVR2B and BMP-3b are members of TGF-Beta superfamily; CXCL5 and MMP13 participate into IL-17 signaling pathway; FGF2 and FGF12 are proteins of FGF family. We are currently performing the transcriptome analysis, and the next step will be to correlate detected proteins with mRNA levels considering also post-transcriptional or epigenetic modifications. Finally we will validate these findings with other quantitative techniques (like ELISA and RT-PCR).

Conclusions: Our first proteomic and broad spectrum analysis suggest the implication of molecular pathways involved in a wide variety of cellular functions such as gene expression modulation, differentiation, angiogenesis, tissue repair, acute and chronic inflammatory response which may explain the beneficial effects of MBT observed in osteoarthritis. This "omic" approach (proteomic and transcriptomic), generated a huge amount of data that is currently under statistical and bioinformatic analysis.

References:

[1] Forestier R, Desfour H, Tessier JM, et al. Spa therapy in the treatment of knee osteoarthritis: a large randomised multicentre trial. Ann Rheum Dis. 2010;69:660-5

Acknowledgements: Supported by Fondazione per la Ricerca Scientifica Termale (FORST) grant.

Disclosure of Interest: None declared

DOI: 10.1136/annrheumdis-2017-eular.6874

\section{SAT0529 PROLONGED TNF-INHIBITOR TREATMENT DURATION IS ASSOCIATED WITH LOWER RISK OF HAND OSTEOARTHRITIS PROGRESSION IN PATIENTS WITH RECENT-ONSET RHEUMATOID ARTHRITIS AFTER 10-YEAR FOLLOW-UP}

M. Loef ${ }^{1}$, F.P. Kroon ${ }^{1}$, S.A. Bergstra ${ }^{1}$, J.A. van der Pol ${ }^{1}$, W.F. Lems ${ }^{2}$

P.J. Kerstens ${ }^{3}$, C.F. Allaart ${ }^{1}$, M. Kloppenburg ${ }^{1,4} .{ }^{1}$ Rheumatology, Leiden University Medical Center, Leiden; ${ }^{2}$ Rheumatology, VU University Medical Center, Amsterdam; ${ }^{3}$ Rheumatology, Westfriesgasthuis, Hoorn; ${ }^{4}$ Epidemiology, Leiden University Medical Center, Leiden, Netherlands

Background: Increasing evidence indicates involvement of the pro-inflammatory cytokine TNF- $\alpha$ in hand osteoarthritis (OA) pathogenesis. Ten years clinical and radiologic follow-up of the BeSt study, a randomized trial primarily designed to investigate targeted treatment in rheumatoid arthritis (RA) patients, offered the unique opportunity to study the long-term effects of TNF inhibitors (TNFi) on the development and progression of hand OA. The distal interphalangeal joints (DIPs) are rarely affected in $\mathrm{RA}$, which allowed to evaluate primary $\mathrm{OA}$ separately. Objectives: To investigate the effect of TNFi on incidental and progressive radiographic hand $\mathrm{OA}$ after 10 -year follow-up in recent-onset RA patients. Methods: At baseline and 10-year follow-up 262 patients (mean age 52 years, $66 \%$ women) were available for radiologic assessment of hand OA. Eighteen interphalangeal joints (IPs) were scored for osteophytes (OP) using the Osteoarthritis Research Society International (OARSI) atlas (0-3; summated score $0-54$ ), and according to the Kellgren-Lawrence (KL) scoring method (0-4; summated score $0-72$ ). Incidental OA was defined as an increase $\geq 1$ in summated OP score or $\geq 2$ in summated $K L$ score in absence of $O A$ at baseline, and progressive $\mathrm{OA}$ as an increase $>3$ in summated $\mathrm{OP}$ or $\mathrm{KL}$ score in presence of $\mathrm{OA}$ at baseline, based on the smallest detectable change. TNFi treatment and disease activity score (DAS) were assessed on standardized visits at a three-monthly interval. Associations between duration of TNFi treatment in months and incidental and progressive OA were analyzed using generalized linear models with Poisson distribution and robust standard errors, while adjusting for age, gender, time averaged DAS, time averaged Sharp-van der Heijde score and hand $O A$ severity at baseline.

Results: Based on the OP score, 126 patients (48\%) were classified with OA at baseline in the DIPs and 82 patients (31\%) in the proximal IPs (PIPs). Incidental OA developed in $33 \%$ of patients in DIPs and in $42 \%$ in PIPs. Progressive OA occurred in $30 \%$ of patients in DIPs and in $38 \%$ in PIPs. Of patients with and

Table 1. Associations between TNFi treatment duration and progressive hand OA in $261^{*}$ patients, defined by osteophyte score and Kellgren-Lawrence score.

\begin{tabular}{|c|c|c|c|}
\hline Joint type & $\begin{array}{c}\text { Number of patients } \\
\text { treated/ median (IQR) } \\
\text { duration of treatment }\end{array}$ & $\begin{array}{c}\text { Adjusted } \\
\operatorname{RR}(95 \% \mathrm{CI})^{\wedge}\end{array}$ & P value \\
\hline \multicolumn{4}{|c|}{ Patients with a $\triangle O P$ score $\geq 3$ units in presence of $O A$ at baseline } \\
\hline DIPS & $67 / 33(12-72)$ & $0.987(0.978-0.996)$ & 0.003 \\
\hline PIPs & $43 / 30(12-72)$ & $0.995(0.985-1.005)$ & 0.321 \\
\hline \multicolumn{4}{|c|}{ Patients with a $\triangle K L$ score $\geq 3$ units in presence of $O A$ at baseline } \\
\hline DIPS & $51 / 39(12-87)$ & $0.991(0.984-0.998)$ & 0.007 \\
\hline PIPs & $32 / 36(12-72)$ & $0.995(0.986-1.004)$ & 0.303 \\
\hline
\end{tabular}

*1 patient missing due to poor quality of hand radiographs at 10 years. ^Adjusted

for age, gender, time averaged DAS, time averaged SHS, severity of OA at baseline without $\mathrm{OA}$ at baseline, irrespective of joint location, $63 \%$ and $55 \%$ were treated with TNFi, with a median treatment duration of 47 and 36 months, respectively. No effect of TNFi treatment duration was seen on incidental hand OA. On progressive hand $\mathrm{OA}$, every month of TNFi treatment resulted in a reduced relative risk (RR) of OA progression in DIPs with a RR (95\% confidence interval) of 0.987 (0.978-0.996), but not in the PIPs. The results from the analyses with the KL scoring method were comparable to the OP score, as shown in table 1.

Conclusions: Prolonged TNFi treatment is associated with a reduced relative risk on radiologic hand OA progression in DIPs, but not in PIPS, after 10 years. Although the effect sizes are small, these results provide evidence for influence of TNF- $\alpha$ in hand OA pathogenesis.

Disclosure of Interest: M. Loef: None declared, F. Kroon: None declared, S. A Bergstra: None declared, J. van der Pol: None declared, W. Lems: None declared, P. Kerstens: None declared, C. Allaart Grant/research support from: The BeSt study was designed by the investigators and supported by a government grant from the Dutch Insurance Companies, with additional funding from Schering-Plough B.V. and Janssen B.V., M. Kloppenburg: None declared

DOI: 10.1136/annrheumdis-2017-eular.2337

\section{SAT0530 CENTRAL SENSITISATION IN HAND OSTEOARTHRITIS: THE ANTERIOR CINGULATE CORTEX IS INVOLVED IN PAIN PROCESSING}

M. Russell ${ }^{1}$, F. Howe ${ }^{2}$, T. Barrick ${ }^{2}$, N. Sofat ${ }^{1} .{ }^{1}$ Institute of Infection \& Immunity; ${ }^{2}$ St George's, University of London, London, United Kingdom

Background: We have previously shown that people suffering with chronic pain in hand OA have activation of central brain pain processing centres localised to the anterior cingulate cortex, insular cortex and thalamus by functional neuroimaging (1) and algometry (2).

Objectives: We hypothesised that hand $O A$ subjects will have structural differences in 3 pain-processing regions of the brain - the anterior cingulate cortex (ACC), insular cortex and thalamus - based on activation of brain centres we have found previously (1). Our brain neuroimaging work was part of a randomised, placebo-controlled trial to assess the effect on clinical pain outcomes by intervention with centrally-acting analgesic agents: pregabalin or duloxetine. Methods: The primary outcome measures for our clinical trial were hand pain Numerical Rating Scale (NRS) and AUSCAN pain after 12 weeks' treatment. In secondary outcome analyses, participants with hand OA $(n=28)$ underwent T1weighted MRI of the brain before and after 12 weeks of treatment with pregabalin, duloxetine or placebo therapy. Grey matter brain structure was compared using FreeSurfer regional volumetric analysis and voxel-based morphometry (VBM) to age-matched controls $(n=11)$, and evaluated for volume changes in the ACC, insular cortex and thalamus.

Results: In our clinical trial, we observed clinically significant improvement in pregabalin, duloxetine and placebo treatment groups after 12 weeks (ANOVA $\mathrm{p}=0.0078$ ). Most notably, pairwise comparisons for pregabalin vs placebo showed significant improvement for NRS pain and AUSCAN pain outcomes in the ITT analysis $(p<0.05)$, but not for duloxetine vs placebo after 12 weeks' $(p>0.05)$. Both voxel-wise and regional volumetric analyses demonstrated areas of reduced grey matter volume in the ACC of hand OA subjects, relative to control subjects, at baseline $(p<0.05)$ (Figure 1). The structural differences in the ACC persisted following 12 weeks of treatment with pregabalin, duloxetine or placebo therapy $(p<0.05)$. We did not observe structural differences in the insular cortex or thalamus in any of the three groups.

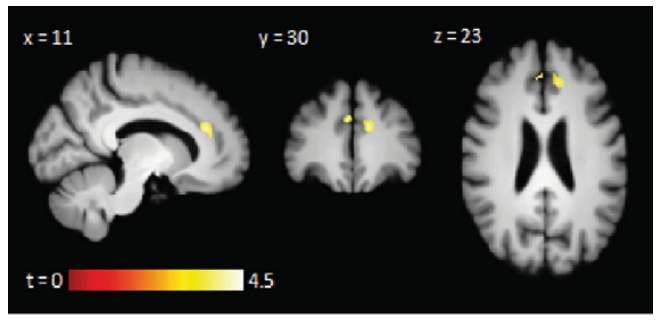

Figure 1. VBM analysis demonstrating clusters of reduced grey matter volume in the $A C C$ in participants with hand $\mathrm{OA}$, relative to control subjects, at baseline. The left side of the figure represents the left side of the brain.

Conclusions: We found that the ACC volume was reduced in participants with hand OA. The ACC is a key pain-processing region of the brain. Changes in ACC grey matter volume have previously been described in other painful conditions, but not hand OA. ACC grey matter volume reduction is thought to represent neural plasticity in chronic pain states. Our data supports the role of central sensitisation in hand $\mathrm{OA}$ and provides a rationale for the further investigation of centrallyacting analgesics in its management. Our trial demonstrated improvement in clinical endpoints for pain for pregabalin vs placebo and duloxetine vs placebo, respectively $(p<0.05)$. However, structural differences in the ACC were still evident following 12 weeks of treatment with pregabalin or duloxetine. This may relate to the relatively short duration of treatment in our study. Alternatively, the baseline differences in the ACC may represent irreversible changes. Longitudinal studies with greater follow-up periods are necessary to further investigate this. 